\title{
Teledermatology protocol for screening of Skin Cancer ${ }^{*}$
}

\author{
Maria Fernanda Piccoli ${ }^{1}$ \\ Harley Miguel Wagner²
}

\author{
Bruna Dücker Bastos Amorim ${ }^{1}$ \\ Daniel Holthausen Nunes ${ }^{3}$
}

\begin{abstract}
BACKGROUND: Telemedicine refers to the use of technology as improvement of healthcare delivery to places where distance becomes an obstacle. Its use represents a great potential for dermatology, a specialty whose visual analysis phase is essential in diagnosis.

OвJестіVEs: To analyze the compatibility index of skin cancer diagnoses between primary care and teledermatology, and to validate a protocol for standardization of digital imaging to obtain the reports in teledermatology. METHODS: An observational cross-sectional study developed through the census of 333 examination requests, received between January/2012 and July/2012, in the Center for Telemedicine and Telehealth of SES-SC. We used a protocol for photographic lesion standardization, consisting of three steps (panoramic photo, close-up with ruler and dermoscopy). After collection, the data were sent to a virtual site on the Internet, and recorded with the use of an electronic health record containing the images, the skin phototype and demographic characteristics.

RESULTS: The level of compatibility between the diagnosis of skin cancer in Santa Catarina's primary care and the diagnosis proposed by teledermatology was $19.02 \%$. Proportionally, it was $21.21 \%$ for BCC, $44.44 \%$ for SCC and $6.98 \%$ for MM. The protocol was statistically significant ( $<<0.05)$, with an OR of 38.77 .

CONCLUSION: The rate of diagnostic compatibility of skin cancer was low and the use of the protocol optimized the chance of validating requests for examination.
\end{abstract}

KEYWORDS: Mass screening; Skin neoplasms; Telemedicine

\section{INTRODUCTION}

It is not difficult to see the potential that the dissemination of use of telemedicine has to offer to the practice of Dermatology. Especially if we consider that, in this branch of medicine, which is responsible for the care of the skin and its adnexa, the diagnosis of patients is often made - with due respect to the patient's medical history - through images and visualizations of the lesion. Thus, one can say that teledermatology corresponds to the branch of telemedicine that seeks to subsume new IT and communication technologies into dermatological practices, in order to reduce the need for face-to-face encounter between the patient and the specialist. Its objective is to ensure effective healthcare planning, research, education, clinical discussion, second opinions and specialized dermatological care. Because dermatology deals with a strong visual component, it is highly benefited by telemedicine, which is basicly used to provide teleconsultation, i.e., remote medical assistance. ${ }^{1-5}$
There are two methods for the exchange of information in Telemedicine which are also used in second formative opinion: the synchronous (realtime) mode, in which the person asking the question and the one answering it, together with the patient, participate in live consultations; and the asynchronous mode (store-and-forward), in which a question is transferred to a database for later consultation. The latter mode is used by the Santa Catarina Telemedicine Network (RCTM, Rede Catarinense de Telemedicina)..$^{1,6-14}$

Due to the large number of dermatological diseases and their clinical and temporal variability, and the difficulty in standardizing photos, the creation of specific protocols and adequate tools for enabling remote diagnostics in dermatology is of paramount importance. ${ }^{1,15-17}$

Currently, it is not difficult to realize that one of the main impediments to the achievement of a comprehensive and effective healthcare system is econom-

Received on 24.09.2013.

Approved by the Advisory Board and accepted for publication on 21.03.2014.

Study conducted at the Center for Telemedicine and Telehealth, State Secretariat of Health, Santa Catarina (SES-SC) - Florianópolis (SC), Brazil.

Financial Support: None

Conflict of Interest: None

Universidade do Sul de Santa Catarina (UNISUL) - Palhoça (SC), Brazil.

Fundação de Ensino e Engenharia de Santa Catarina (FEESC) - Florianópolis (SC), Brazil.

Universidade Federal de Santa Catarina (UFSC) - Florianópolis (SC), Brazil. 
ic. In this sense, second formative opinion and telediagnosis offer alternatives to overcome this barrier, since they are considered to be low-cost procedures, when compared to the concrete results achieved. ${ }^{1,14}$

Another remarkable fact is the maldistribution of physicians in the state of Santa Catarina Most professionals, for many different reasons, tend to establish themselves around urban centers, often leaving the outlying areas under the responsibility of non-specialist professionals. In this context, the second formative opinion is used with the objective of assessing the need for referral of a patient to a specialist away from home. This prevents that simple therapeutic treatments are put off due to the distance between patient and specialist. Furthermore, this new technique for exchange of information allows the provision of adequate treatment to residents of remote regions. . $^{1,3,12,17,18}$

An accurate diagnosis is crucial for a successful therapeutic intervention. However, the dynamics of medicine as well as the various ways in which a disease can manifest itself interferes with the art of clinical reasoning. Given this reality, added to the economic and geographic factors outlined above, telediagnosis through teledermatology appears as an innovative method of professional aid, essential to enabling proper access to health.

In this perspective, the verification of the effectiveness of telediagnosis in dermatology proves extremely valuable for the consolidation of this new technique as an alternative to achieve a democratic and specialized medical treatment.

Thus, this study aims to determine the diagnostic compatibility between general practitioners of primary healthcare services and telediagnosis in dermatology by a specialist physicians. This should be done by using a structured protocol of teledermatology for the diagnosis of the main types of skin cancer that affect the population of Santa Catarina.

\section{METHODS}

This is an epidemiological case-control study to validate the structured protocol of teledermatology for the diagnostic screening of skin cancer. A descriptive cross-sectional approach was adopted for the independent variables. The study was conducted at the Center for Telemedicine and Telehealth of the State Secretariat of Health of Santa Catarina.

The study population included patients treated in Basic Healthcare Units (BHU) of Santa Catarina who sought treatment due to visible dermatological complaints.

The physician who required the examination (assistant - non-specialist physician), responsible for on-site consultations in Primary Health Care (PHC), and the dermatologist responsible for remotely mak- ing the analysis and proposing a diagnosis based on the photos of the lesions (telediagnosis) also participated in the study .

We used the census method to select the study population.

\section{Inclusion and exclusion criteria}

All patients - - regardless of age, gender, socioeconomic status, skin type or origin - who sought a BHU in Santa Catarina with visible dermatological complaints and after initial medical assessment with a nonspecialist were referred for consultation with a dermatologist (specialist) via telediagnosis were admitted to this study. However, only those who signed the informed consent form participated in the study.

\section{Groups}

Cases: patients who presented with suspect lesions detected by the attending primary care physician.

Controls: patients who had lesions compatible with skin cancer according to the specialist's assessment.

Exclusion criteria were: patients who attended the consultation with dermatological complaints, but had no visible lesions or who had lesions which presented insufficient quality for evaluation or patients who were undergoing dermatological treatment and presented only clinical symptoms without visible dermatological symptoms. All those who did not sign the consent form were also excluded from the study.

\section{Data collection}

Aiming at the standardization of procedures for the collection of data for this study, which occurred by means of digital imaging, we provided trainings to the professionals working at the nine healthcare centers which are evenly distributed among the macroregions that make up the State of Santa Catarina. This space was called Qualified Healthcare Units for the Collection of Tests and counted with the participation of professional technicians, previously trained to obtaining adequate images.

\section{Procedures}

During on-site consultation with a non-specialist physician in the Basic Healthcare Units, all relevant patient data were entered into an electronic health record. This made a referral to a specialist possible, when needed. Patients who had signs or symptoms that suggested skin cancer had indication of referral.

Those patients who were selected for a consultation with a dermatologist were conducted to the nearest Qualified Healthcare Unit for the Collection of Tests, where data collection was conducted once the consent form had been signed.

The photographic record for the investigation 
of suspected cancer cases was performed in three steps: Panoramic Photo, Close-up with scale and Dermoscopy.

Regarding the Panoramic Photo, the technician was instructed to identify each lesion using a label with the date, patient's initials and lesion number. He also was told to make a panoramic image of the region of the body affected by one or more lesions (head, torso, arms and legs, either front or back). At this stage, the technician was instructed not to use the zoom function.

The second step consisted of close-up with scale. The technician was instructed to take the picture at a distance of about thirty centimeters from the lesion, without using the zoom function, and to place a scale parallel to the lesion in order to serve as a reference of the actual size of the lesion.

Finally, the third photo was taken by using dermoscopy. The dermatoscope was connected to the digital camera. In this step, the technician applied gel on the lens of the camera and photographed each lesion by using the dermatoscope placed against the patient's skin and the maximum zooming level.

After collection, the data were transmitted through an environment developed exclusively for sending data corresponding to each patient on the Internet. Whenever a new case arrived, a message was sent to the participating physician (specialist), communicating the new remote consultation request, which was accessed through a restricted site.

The doctor in charge would show his diagnosis autonomously, respecting the seventy-two-hour deadline and using the clinical photographs and medical data sent by the non-specialist physician.

\section{Data collection instrument}

A protocol was used as an instrument for data collection. It included information relating to the procedure used for obtaining images of the lesions and the anatomical region affected. After the protocol was completed, the photos were sent via the Internet to a restricted virtual site, to which the remote specialist (dermatologist) and the on-site non-specialist physician had access.

The collection of images was performed by using Cyber Shot cameras, since photographs of lesions are part of the basic data necessary for conducting telediagnosis. These images were then uploaded onto the computer via USB cable and stored in JPEG format.

\section{Statistical analysis}

Data were analyzed using the SPSS 18.0 program. Sensitivity and specificity as well as PPV and NPV, and accuracy of the diagnosis of each tumor type were calculated. The odds that the medical expert could make a diagnosis for the dermatological examination sent, following the established protocol or not, were calculated by the odds ratio, considering the $95 \%$ confidence interval and $p<0.05$. For validation, methods were calculated for agreement using the kappa coefficient $(\boldsymbol{\kappa})$.

\section{Ethical aspects}

This study is in accordance with the guidelines and rules for research involving human subjects (resolution 196/1996 of the National Health Council) and was approved by the research ethics committee of UNISUL, Opinion No. 11.190.04.01).

All patients who agreed to participate in the study signed an informed consent form in duplicate. Both the case histories and the photographs were performed on a secrecy basis. ${ }^{19}$

\section{RESULTS}

The analyzed group consisted of 333 requests for evaluation. Of these, 149 were invalidated for various reasons, such as: trial examination; repeated examination, examination not in accordance with the protocol and examination with image out of focus, as shown in table 1.

With regard to examinations not in accordance with the protocol being a reason for invalidation, one hundred twenty-eight such episodes were reported between the months of January and July, accounting for $85.91 \%$ of the total number of examinations. The incidence images out of focus caused the invalidation of twelve cases $-8.05 \%$ of the total number of invalidated examinations.

149 cases received from the primary healthcare network were invalidated. This occurred most frequently in January and accounted for fifty cases $(33.55 \%)$ and least frequently in July (3.35\%). Likewise, the most prevalent reason for invalidation of diagnostic hypotheses was due to examinations which were not performed in accordance with the protocol and the least prevalent reason was due to trial examinations, according to the data cited above.

Regarding the remaining group - 184 evaluation requests - we found a higher frequency of females (135 $73.37 \%$ ). The hegemonic skin color was white. Phototype III was evidenced in 135 patients (55.97\%). Phototype II in 30 patients $(16.84 \%)$ and phototype I in 19 patients $(10.86 \%)$. The average age was $54.74( \pm 15.17)$ years.

Furthermore, we analyzed the compatibility of diagnoses for each type of skin neoplasia, according to table 2, itemized below.

Among the analyzed group, $71.74 \%$ were referred with suspected basal cell carcinoma (BCC), $4.89 \%$ with suspected squamous cell carcinoma (SCC) 
TABLE 1: Outcome of treated lesions based on location

\begin{tabular}{|c|c|c|c|c|c|c|c|c|}
\hline \multirow[t]{2}{*}{ Invalidation Reason } & \multicolumn{5}{|c|}{ Number of invalidations per month } & \multirow[b]{2}{*}{ Jun } & \multirow[b]{2}{*}{ Jul } & \multirow{2}{*}{$\begin{array}{l}\text { Total } \\
\text { n }(\%)\end{array}$} \\
\hline & Jan & Feb & Mar & Apr & May & & & \\
\hline Trial examination & 0 & 0 & 3 & 0 & 0 & 0 & 0 & $3(2.01)$ \\
\hline Repeated examination & 3 & 0 & 3 & 0 & 0 & 0 & 0 & $6(4.03)$ \\
\hline $\begin{array}{l}\text { Examination conducted } \\
\text { not in accordance with } \\
\text { the protocol }\end{array}$ & 47 & 5 & 30 & 14 & 18 & 9 & 5 & $128(85.9)$ \\
\hline $\begin{array}{l}\text { Examination with image } \\
\text { out of focus }\end{array}$ & 0 & 5 & 4 & 0 & 2 & 1 & 0 & $12(8.05)$ \\
\hline Total & 50 & 10 & 40 & 14 & 20 & 10 & 5 & 149 (100) \\
\hline
\end{tabular}

$\mathrm{n}$ - absolute number

TABLE 2: Compatibility among different skin neoplasias according to suspicion and diagnostic hypothesis

\begin{tabular}{|c|c|c|c|}
\hline \multirow[t]{2}{*}{ Types of skin cancer } & \multicolumn{3}{|c|}{ Compatibility } \\
\hline & $\begin{array}{l}\text { Yes } \\
\text { n (\%) }\end{array}$ & $\begin{array}{l}\text { No } \\
\text { n (\%) }\end{array}$ & $\begin{array}{l}\text { Total } \\
\text { n (\%) }\end{array}$ \\
\hline \multicolumn{4}{|l|}{ Basal cell cancer (BCC) } \\
\hline BCC & $28(21.2)$ & $104(78.8)$ & $132(71.7)$ \\
\hline Other skin cancers & $8(15.4)$ & $44(84.6)$ & $52(28.3)$ \\
\hline Total & $36(19.6)$ & $148(80.4)$ & $184(100)$ \\
\hline \multicolumn{4}{|c|}{ squamous cell carcinoma (SCC) } \\
\hline SCC & $4(44.44)$ & $5(55.56)$ & $9(4.89)$ \\
\hline Other skin cancers & $9(5.14)$ & $166(94.8)$ & $175(95.1)$ \\
\hline Total & $13(7.07)$ & $171(92.9)$ & $184(100)$ \\
\hline \multicolumn{4}{|c|}{ Malignant melanoma (MM) } \\
\hline MM & $3(6.98)$ & $40(93.02)$ & $43(23.4)$ \\
\hline Other skin cancers & $1(0.71)$ & $140(99.3)$ & $141(76.6)$ \\
\hline Total & $4(2.17)$ & $180(97.8)$ & $184(100)$ \\
\hline
\end{tabular}

and $23.37 \%$ with suspected malignant melanoma (MM).

Regarding BCC, $19.57 \%$ of the cases analyzed by teledermatology obtained this neoplasia as a diagnostic hypothesis. Of these, $15.38 \%$ were diagnosed as other skin cancers, while $21.21 \%$ confirmed the suspicion received from the primary healthcare unit. On the other hand, of the 132 cases sent to telediagnosis as suspected BCC, $78.79 \%$ were not compatible with the diagnosis proposed by the specialist and of 52 referrals diagnosed as non-BCC carcinomas, $84.62 \%$ agreed with the dermatologist's opinion.

In the case of SCC, of all valid examinations, $7.07 \%$ were confirmed by the specialist as such. $5.14 \%$ of these were derived from the primary healthcare system as other skin cancers and $44.44 \%$ as suspected SCC. However, of 9 examinations coming as SCC, $55.56 \%$ were incompatible with the proposed diagno- sis made by the telemedicine physician.

With regard to MM, only $2.17 \%$ were classified as such by the dermatologist. Of these, $0.71 \%$ were diagnosed as other skin cancers, while $6.98 \%$ confirmed the suspected diagnosis of primary care. However, of the 43 suspected cases of MM, 93.02\% showed no compatibility.

After checking the compatibility between suspicion and diagnostic hypothesis, we analyzed the percentage of diagnostic examinations according to each type of skin cancer, namely: sensitivity, specificity, accuracy, positive predictive value and negative predictive value, as shown in table 3.

Sensitivity for the diagnosis of BCC was $77.77 \%$. This means that, among the 36 cases qualified by teledermatology as BCC, 28 were sent to telediagnosis with this suspicion, whereas 8 cases were diagnosed by the specialist as BCC, although they had been referred to as other skin cancers.

Specificity in regard to the BCC was $29.73 \%$ Therefore, of the 148 cases so classified by the specialist, only 44 were sent as being other skin cancers.

$39.13 \%$ of accuracy was obtained in the diagnosis of BCC. Therefore, 28 suspected cases of BCC were compatible with the teledermatology diagnosis, while 44 cases of suspected other skin cancers (non-basal cell cancers) were also compatible. Both accounted for a total of 184 tests performed.

With regard to SCC, diagnostic sensitivity was $30.77 \%$. Thus, among the 13 cases classified as SCC by the specialist, 4 had been sent by primary care with such suspecion, while 9 had been sent as other skin cancers, not SCC.

TABLE 3: Percentage of diagnostic tests applied to each type of skin cancer, according to the examination requested by the primary healthcare network

\begin{tabular}{llllll}
\hline & \multicolumn{5}{c}{ Diagnostic tests } \\
\cline { 2 - 5 } Skin neoplasias & Sensibility (\%) & Specificity $(\%)$ & Accuracy (\%) & PPV (\%) & NPV (\%) \\
\hline Basal cell cancer (BCC) & 77.7 & 29.7 & 39.1 & 21.2 & 84.6 \\
squamous cell carcinoma (SCC) & 30.7 & 97.1 & 92.4 & 44.4 & 94.8 \\
Malignant melanoma (MM) & 75.0 & 77.7 & 77.7 & 6.98 & 99.3 \\
\hline
\end{tabular}

PPV - positive predictive value; NPV - negative predictive value 
SCC specificity was $97.07 \%$. Therefore, of the 171 cases which did not have such a diagnostic proposal, 166 were suspected to be other skin cancers

The accuracy of SCC diagnosis was $92.39 \%$. From a total of 184 examinations performed, 4 suspected SCC cases presented diagnostic compatibility, whereas 166 suspected cases of other skin cancers (not SCC) were also compatible.

Regarding MM, the diagnostic sensitivity was $75 \%$. That is, among the 4 cases classified as MM, 3 were sent to telediagnosis with such suspicion, while 1 was described by the teledermatology physician as $\mathrm{MM}$, although it had been classified as other skin cancer by the non-specialist physician.

MM specificity was $77.77 \%$. Thus, of the 180 cases presented by telediagnosis as MM, 140 had been suspected as other skin cancers, not MM.

The accuracy of the diagnosis of MM was $77.70 \%$. Therefore, 3 suspected MM cases were consistent with the specialist's opinion, while 140 examinations with suspecion of other neoplasias were also compatible. A total of 184 examinations were conducted.

Regarding the PPV of BCC, we obtained a value of $21.21 \%$. This means that, of the 132 examinations referred to the specialist as suspected BCC, 28 were compatible. The negative predictive value of BCC was $84.62 \%$. Of 52 examinations sent by the primary care system as other skin cancers, 44 were classified as non-BCC by the dermatologist.

VPP of SCC was found to be $44.44 \%$. Of the 9 examinations sent by primary care as suspected SCC, 4 were consistent with the diagnosis made by the specialist. NPV of CEC was $94.86 \%$, since of 175 reports sent to telediagnosis as other skin neoplasias, 166 were not considered by the specialist as SCC.

PPV of MM was found to be $6.97 \%$. Of the 43 examinations referred to as malignant melanoma, only 3 were consistent with the diagnosis proposed by the specialist. In contrast, the NPV of MM was $99.29 \%$. Of the 141 examinations referred to telediagnosis as MM, 140 showed diagnostic compatibility.

Regarding the use of the protocol for the standardization of digital imaging obtention, this study found that, of the 333 requests generated in the primary healthcare network, 180 examinations indicated that the protocol had been followed appropriately, and among these, 159 were considered suitable to be sent to the specialist for evaluation. Conversely, 153 requests indicated some problem in compliance with the protocol. Of these, only 25 could be analyzed.

The odds calculated for the dermatologist to make the teledermatology diagnosis following the protocol correctly, was OR 38.77 (95\% CI, 20.74 to 72.43; p $<0.001)$, showing a substantial agreement $(\boldsymbol{k}=0.69)$.

\section{DISCUSSION}

This study was conducted in the search for alternatives to ensure proper access to health care and in partnership with non-specialist doctors from different macro-regions of Santa Catarina. Its aim was to analyze the compatibility index between the suspected diagnosis of skin cancer made by basic care, and the diagnosis proposed by teledermatology. Moreover, we aimed at validating a protocol for standardizing the obtention of digital images for telediagnosis reports in dermatology.

Currently, CFM Resolution 1.643/2002 defines and regulates the provision of services through telemedicine. Telediagnosis in Dermatology is not yet regulated in the Unified Healthcare System (SUS). Santa Catarina is a pioneer in the use of teledermatology. However, in order to guarantee the long-term sustainability of the model, it has to be regulated by CONITEC (National Commission for Technology Incorporation in the SUS), a body of the ANVISA (National Health Surveillance Agency).

This is a unique study that seeks to assist in the improvement of the teledermatology system in the State of Santa Catarina. Methodological efforts to legitimize the results were undertaken.

First, the sampling census design used in this study assured a valid number of participants to be included in the investigation. Second, the quality of data collection was assured by the standardization of procedures, which was achieved through the provision of trainings to the professionals of healthcare centers in the state who were performing the tests, and through the technology employed - an environment exclusively created on the Internet for sending patients' data. Third, the use of protocols as an instrument for objective data collection, together with predefined procedures for the performance of photographs of skin lesions, avoids vulnerabilities as interpretation biases, confirming the verification of adequate diagnosis. Fourth, the careful application of the methodology generated 149 invalidations, leaving only 184 requests to be analyzed.

Finally, the fact that the research was conducted at the Center for Telemedicine and Telehealth of the State Secretariat of Health was one of the strengths of conducting this study, since the above-mentioned environment is a reference environment in terms of telemedicine in Santa Catarina.

In contrast, the recent implementation of teledermatology care in the state, parallel to the fact that we depend on a virtual environment software, caused practical problems to the system. In addtion, we had to deal with the inexperience of the technical teams (even after training was provided), and with difficul- 
ties in the operation of the virtual program, which resulted in a significant reduction in the total number of requests sent to telediagnostics.

Regarding the number of invalidations, we found that the highest rates occurred in the first months. This result may be associated with the fact that the system had been recently implemented, since invalidations gradually reduced in the subsequent months, indicating the adaptation of the team and their learning of the technique. Moreover, it could mean that these figures will decrease even more with time. Corroborating the hypothesis, it is noteworthy that the main reason for invalidation was due to sending examinations which were not conducted in accordance with the protocol, i.e., examinations whose annulment occurred due to incorrect use of photography techniques and/or data completion. The invalidation rate later possibly reduced because the team acquired more experience with the procedures.

Conversely, although in the early phase of implementation of the system, the month of February showed low rates of invalidations. This can be explained by the lower amount of requests analyzed by the specialist physician in these period.

In the case of the remaining group, the majority of subjects were female. This result may be linked to a higher demand for primary care services by this population. Such supposition is supported by Figueiredo and Pinheiro et al, who argue that women are more concerned with their personal health. ${ }^{20,21}$ Another possibility may be related to the higher incidence of skin cancer in the female population, since, according to Azulay et al, BCC - which accounts for $70 \%$ of cases - and MM are slightly more frequent in women. ${ }^{22}$

Most of the cases studied were white persons (83.67\%), including phototypes I, II and III, a factor that most likely is related to the higher incidence of skin cancer in people with lighter skin color. This hypothesis is based on the literature, which supports the direct relationship between skin cancer and skin color, in the sense that the lighter the skin color, the greater the frequency of occurrence of skin cancer. ${ }^{22-28}$ Nevertheless, another plausible explanation for the high incidence of white people in the study is due to the fact that the population of Santa Catarina is predominantly light-skinned (84\%), according to IBGE. ${ }^{29}$

As to the age group, the average age of subjects was 54.9 years, which corroborates the data found by other authors, like Ignatius et al and Nasser. ${ }^{30,31}$ This can be explained by the cumulative action of ultraviolet radiation on the skin over the years, a significant risk factor for the development of skin cancer, according to the authors mentioned before.

Turning to the diagnostic suspicions sent by the primary care network, we found that most referrals $(71.74 \%)$ were suspicions of BCC. One possible explanation for this is the high prevalence of this type of cancer in the population, as studies by Azulay et al, Chinem \& Miot and the National Cancer Institute (INCA) report. These studies state that BCC corresponds to $70 \%$ of all skin neoplasias. ${ }^{22,32,33}$ Moreover, the fact that this type of cancer is considered the most frequent in the literature may lead non-specialists to generalize suspected cases in the case of diagnostic doubt, also increasing the number of referrals due to BCC.

With respect to the SCC, the total number of referrals, compared to BCC and MM, was significantly lower $(4.89 \%)$, which confirms the epidemiology found in the literature, according to studies by Azulay et al., Nasser and Nunes et al. ${ }^{22,32,34}$ This can be explained by the difficulty faced by the non-specialist physician in distinguishing between BCC and SCC, as they are considered as one another's main differential diagnoses, according to Azulay et al. ${ }^{22}$ Moreover, as already said before, when uncertain about the diagnosis, the general practitioner may have opted for the one known to be more common, which possibly reduced the incidence of suspected SCC. Likewise, the fact that the incidence of suspected MM in this study does not agree with the literature, may result in significant reduction of referrals due to SCC, since the INCA and Dimatos et $\mathrm{a}^{13,35}$ state that the frequency of melanoma accounts for around $4 \%$, while in the present study $23.37 \%$ of the reports were sent as suspected MM.

Thus, with respect to MM, as already discussed above, the number of referrals was incongruent with the current literature. ${ }^{33,35}$ This possibly occurred due to the difficulty of the non-specialist physician in distinguishing between malignant lesions and lesions with benign features, given that $\mathrm{MM}$ is a tumor originating from melanocytes which develops, in most cases, from a preexisting nevus, i.e., possibly even before a nevus with changes that do not characterize malignancy, the general practitioner classifies it as MM. ${ }^{22}$ However, sensitivity was found to be $75 \%$, which is a reasonable value. This information deserves further reflection, though, since it should be taken into account that the high number of referrals as suspected $\mathrm{MM}$, in association with the low prevalence of the disease, increases the ods of diagnostic compatibility. Likewise, MM specificity may be related to this hypothesis, since in 40 cases of suspected MM, no compatibility was observed.

With regard to diagnostic tests applied to BCC, the sensitivity found denotes that generalist physicians were able to identify most of the cases thus diagnosed by the specialist. This means that the general practitioner would be able to identify this neoplasia in patients. However, this data cannot be interpreted in 
isolation, given that, in terms of specificity, the value found was very low, suggesting that these professionals do not demonstrate the ability to exclude the possibility of BCC when the patient does not have this condition. According to the specialist, this possibly occurred because the generalist is able to make referrals when faced with a suspicious lesion, but fails to recognize the condition.

Regarding the accuracy for BCC, the present study demonstrated that the level of compatibility between the specialist and the non-specialist physicians was low. It is important to highlight that in 104 cases for which the medical expert dismissed the diagnosis of $\mathrm{BCC}$, the non-specialist physician did not disregard the condition, but, on the contrary, he suggested its existence. This fact proves to be extremely important, since this is a condition whose diagnosis is a determining factor in the treatment and cure of the patient.

With respect to the sensitivity found for SCC, we observed a greater difficulty of general practitioners in identifying this disease. Such an event may be related to the lack of specific knowledge in for distinguishing among skin neoplasias. This leads non-specialists, in case of diagnostic doubt, to opt for the more frequent neoplasia that belongs to their differential diagnosis, such as BCC. However, specificity values for SCC were very high, perhaps because of a lack of knowledge about the disease, which provides the non-specialist physician with a greater possibility of diagnostic exclusion than inclusion. However, this information deserves some notes, because although the specificity of SCC was found to be quite high, it is relevant to mention that we found a low absolute number of requests generated as suspected SCC. This does not correlate with the studied literature - which expects a prevalence of around $20 \%$. This may give rise to increased cases of $\mathrm{BCC}$ and/or MM, and thus increase the supposed correctness of the non-specialist physician with regard to non-SCC cases, resulting in a high specificity. ${ }^{22,23,26}$

Moreover, with respect to SCC, the accuracy score obtained can be considered high, and the highest among the types of cancer studied. This is possibly due to the high level of specificity founf for SCC, whose probable cause has already been analyzed.

Regarding the use of the protocol as a method for collecting digital images, we found that, when correct, $88.33 \%$ of the referrals were considered valid and could thus be evaluated by a specialist. Likewise, the absence of appropriate protocol generated only $16.34 \%$ of analyzable requests.

In addition, we highlight the possibility of some of the tests being considered suitable for diagnosis by specialists (although they had not correctly followed the protocol) due to the low complexity and/or typical presentation of the lesion, which is in accordance to the literature. ${ }^{19}$ Thus, when faced with high complexity lesions or lesions without typical features, the use of a structured standard protocol is even more relevant, in order to provide the remote physician with the most efficient tools for making the diagnosis.

These facts, associated with the Odds ratio values found - which showed that the odds of obtaining a diagnosis from a specialist was 38.77 times higher among valid tests, when compared to all invalid tests - suggest that the use of the protocol for obtaining images for telediagnosis may be able to increase the number of tests that the dermatologist can offer, since, according to the literature, the technical quality of the photos is a determining factor in the level of diagnostic accuracy and interobserver agreement. ${ }^{1,16,17}$ This is because the protocol includes: a panoramic photo, which shows the lesion and the affected anatomical region without using the zoom function, enabling the comparison of the lesion size with the patient's body size; a close-up photo with a scale, which allows a close to ideal viewing of the lesion, similar to the faceto-face dermatological examination, besides enabling the verification of the actual size of the lesion; and dermoscopy, which provides key data for the diagnosis of melanocytic lesions (melanoma and benign melanocytic lesions), as well as an approximate analysis of structures present in non-melanoma skin cancers. These steps contribute to ensure the technical quality of the photo, making it suitable for evaluation.

Moreover, the fact that the correlation between the on-side diagnosis and the diagnosis made by teledermatology, when obtained by dermatologists, varies between 80.5 and $91.6 \% .{ }^{6,17}$ However, in these studies, the images were not obtained through a structured protocol and yet the agreement found was high. This way, it is understood that the use of a standard protocol such as the one used in this study may further improve the compatibility of telediagnosis, either among experts or not, thus consolidating teledermatology as a skilled method for diagnosing skin lesions.

A limitation of the study was that the analysis of all images was performed by a single specialist. However, the diagnostic accuracy of dermatologists was not being evaluated, but rather the possibility of a lesion being or not compatible with skin cancer. Thus, sensitivity is more important than specificity in the analysis of images. Thus all doubtful images were considered compatible.

Due to the problems found during the conduction of this study, which resulted in several invalidations, this research gave rise to changes in the collection and submission of test requests, thus contributing to the improvement of teledermatology in Santa Catarina. 
For all that, this research demonstrates the importance of using teledermatology as a modern tool of access to health, since it is a diagnostic method that enables early detection of skin cancer, thus decreasing the number of unnecessary referrals to a dermatologist. Therefore, it helps not only to reduce the waiting time for face-to-face consultations, but also the costs associated with this process.

In addition, the implementation of the teledermatology system in Santa Catarina deserves to be expanded within the context of public policies to provide health access, because it has shown to be a feasi- ble way to address the lack of medical specialists in the public healthcare network, especially in more remote locations. It can be used not only as dermatological care, but also as a method of health planning, research, education and clinical discussion.

\section{CONCLUSION}

The application of a structured protocol was found to be statistically significant, increasing by 38.77 times the odds that examinations were considered suitable for evaluation by the specialist, when correctly completed. 


\section{REFERENCES}

1. Miot HA, Paixão MP, Wen CL. Teledermatology - Past, present and future. An Bras Dermatol. 2005;80:523-32.

2. Romero G, Garrido JA, García-Arpa M. Telemedicineandteledermatology (I): conceptsand applications. Actas Dermosifiliogr. 2008;99:506-22.

3. High WA, Houston MS, Calobrisi SD, Drage LA, McEvoy MT. Assessment of the accuracy of low-cost store-and-forward teledermatology consultation. J Am Acad Dermatol. 2000; $42: 776-83$

4. Warshaw EM, Hillman YJ, Greer NL, Hagel EM, MacDonald R, Rutks IR, et al. Teledermatology for diagnosis and management of skin conditions: A systematic review . J Am Acad Dermatol. 2011;64:759-72.

5. Lozzi GP, Soyer HP, Massone C, Micantonio T, Kraenke B, Fargnoli MC, et al. The additive value of second opinion teleconsulting in the management of patients with challenging inflammatory, neoplastic skin diseases: a best practice model in dermatology? J Eur Acad Dermatol Venereol. 2007;21:30-4.

6. Ribas J, Cunha MGS, Schettini APM, Ribas CBR. Agreement between dermatological diagnoses made by live examination compared to analysis of digital images. An Bras Dermatol. 2010;85:441-7.

7. Wen CL. Telemedicina e Telessaúde. Um panorama do Brasil. Informática Pub. 2008;10:7-15.

8. Bonnardot L, Rainis R. Store-and-forward telemedicine for doctors working in remote areas. J Telemed Telecare. 2009:15:1-6.

9. Kanthraj GR, Srinivas CR. Store and forward teledermatology. Indian J Dermatol Venereol Leprol. 2007;73:5-12.

10. Telemedicina.ufsc.br [Internet] Programa de Telemedicina do Estado de Santa Catarina [acesso 13 Nov 2010]. Disponível em: https://www.telemedicina.ufsc.br/rctm/.

11. Eedy DJ, Wootton R. Teledermatology: a review. Br J Dermatol. 2001;144:696-707

12. D'Elia PB, Fisher PD, Bordin R, Harzheim E, Ramos MC. Agreement between dermatological diagnoses made by direct observation and digital images. An Bras Dermatol. 2007;82:521-7.

13. Bowns IR, Collins K, Walters SJ, McDonagh AJ. Telemedicine in dermatology: a randomised controlled trial. Health Technol Assess. 2006;10:iii-iv, ix-xi,1-39.

14. Whited JD. Teledermatology: Current Status and Future Directions. Am J Clin Dermatol. 2001;2:59-64.

15. Miot HA, Paixão MP, Paschoal FM. Basics of digital photography in Dermatology. An Bras. Dermatol. 2006;81:174-80

16. Levin YS, Warshaw EM. Teledermatology: A Review of Reliability and Accuracy of Diagnosis and Management. Dermatol Clin. 2009;27:163-76, vii.

17. Miot HA. Desenvolvimento e sistematização da interconsulta dermatológica à distância [tese]. São Paulo: Universidade de São Paulo; 2005.

18. Silva CS, Souza MB, Duque IA, de Medeiros LM, Melo NR, Araújo Cde A, et al. Teledermatologia: correlação diagnóstica em serviço primário de saúde. An Bras Dermatol. 2009;84:489-93.

19. Conselho.saude.gov [Internet]. Ministério da Saúde. Conselho Nacional de Saúde. Resolução 196/96 - Diretrizes e normas regulamentadoras de pesquisas envolvendo seres humanos. [Acesso 16 Maio 2011]. Disponível em: http://conselho.saude.gov.br/resolucoes/reso_96.htm

20. Figueiredo W. Assistência à saúde dos homens: um desafio para os serviços de atenção primária. CiênC Saúde Colet. 2005;10:105-9.

21. Pinheiro RS, Viacava F, Travassos C, Brito AS. Gênero, morbidade, acesso e utilização de serviços de saúde no Brasil. Cien Saude Colet. 2002;7:687-707.

22. Azulay, RD, Azulay DR, Azulay-Abulafia L. Dermatologia. 5. ed. Rio de Janeiro: Guanabara Koogan; 2011.
23. Pollock RE, Doroshow JH, Khyat D, Nakao A, O'Sullivan B. UICC Manual de oncologia clínica. 8. ed. São Paulo: Fundação Oncocentro de São Paulo; 2006.

24. Kasper DL, Fauco AS, Longo DL, Braunwald E, Hauser SL, Jameson JL. Harrison medicina interna. 16. ed. Rio de Janeiro: McGraw-Hill; 2006

25. Lopes AC, Neto VA. Tratado de clínica medica. São Paulo: Roca; 2006.

26. Sampaio SAP, Rivitti EA. Dermatologia. 3. ed. São Paulo: Artes Médicas; 2007

27. Goldman L, Ausiello D. Cecil medicine, 23. ed. Rio de Janeiro: Elsevier; 2009.

28. Sachdeva S. Fitzpatrick skin typing: Applications in dermatology. Indian J Dermato Venereol Leprol. 2009;75:93-6.

29. Ibge.gov [Internet]. Instituto Brasileiro de Geografia e Estatística - IBGE. [Acesso em 2012 Set 22] Disponivel em: www.ibge.gov.br/home/presidencia/noticias/noticia_visualiza.php?id_noticia=2170\&i d pagina $=1$.

30. Inácio FD, Becker TCA, Baptista F, Soave JS, Weiler MA, Macena MA et al. Perfil epidemiológico dos portadores de câncer de pele da comunidade dos municípios da região de Campo Mourão - Paraná no ano de 2008. Sábios: Rev Saúde e Biol. 2010;5:51-58.

31. Nasser N. Epidemiologia dos carcinomas basocelulares em Blumenau, SC, Brasil, de 1980 a 1999. An Bras Dermatol. 2005:80:363-68.

32. Chinem VP, Miot HA. Epidemiologia do carcinoma basocelular. An Bras Dermatol. 2011;86:292-305.

33. Inca.gov [Internet]. Instituto Nacional de Câncer - INCA. Pele não melanoma. [acesso 22 Set 2012]. Disponível em: www2.inca.gov.br/wps/wcm/connect/tiposdecancer/site/home/pele_nao_melanoma

34. Nunes DH, Back L, Silva RV, Medeiros VS. Incidence of squamous cell carcinoma of the skin in the city of Tubarao (SC)--Brazil in the years 2000, 2003, and 2006. An Bras Dermatol. 2009;84:482-8.

35. Dimatos DC, Duarte FO, Machado RS, Vieira JV, Vasconcellos ZAA, Bins-Ely J, Neves RD. Melanoma cutâneo no Brasil. Arq Catarin Med. 2009;38:14-9.

How to cite this article: Piccoli MF, Amorim BDA, Wagner HM, Nunes DH. Teledermatology protocol for screening of Skin Cancer. An Bras Dermatol. 2015;90(2):202-10. 